Yasuko Kawauchi MD, * Tsutomu Oshima MD PhD, $†$ Satomi Suzuki MD, * Yuhji Saitoh MD PhD, * Hidenori Toyooka MD PhD‡

\section{Advancement of the mandible facilitates nasal breathing in human subjects sedated with midazolam}

Purpose: To determine how anterior advancement of the mandible (ADM) affects spontaneous breathing through the nasal route in healthy human volunteers sedated with intravenous midazolam.

Methods: In four subjects who exhibited nasal breathing during midazolam sedation (intravenous dose: $0.09 \pm$ $0.02 \mathrm{mg} \cdot \mathrm{kg}^{-1}$, mean $\pm \mathrm{SD}$ ), we measured respiratory rate $(\mathrm{RR})$, peak nasal inspiratory airflow rate $\left(\dot{V}_{\text {nlpeak }}\right)$, peak nasal expiratory airflow rate $\left(\dot{\vee}_{n \text { Epeak }}\right)$, duty ratio (Ti/Ttot) and nasal resistance (Rn) before and after ADM. Nasal resistance was calculated by dividing the difference between nasal mask and oropharyngeal pressure by airflow rate at peak nasal inspiratory airflow.

Results: The RR, $\dot{V}_{\text {nlpeak }}$ and $\dot{V}_{\text {nEpeak }}$ increased following $\operatorname{ADM}(P<0.00 \mathrm{I}$, respectively). On the contrary, Ti/Ttot decreased after ADM $(P<0.001)$. Consequently, ADM decreased Rn from $30.4 \pm 40.8$ to $5.0 \pm 5.6$ $\left(\mathrm{cmH}_{2} \mathrm{O} \cdot \cdot^{-1} \cdot \mathrm{sec}^{-1}\right)$ (mean $\left.\pm \mathrm{SD}\right)(P<0.00 \mathrm{I})$. In these four subjects, no respiratory airflow was observed through the oral route before and after ADM.

Conclusion: Advancement of the mandible decreases nasal resistance, thereby facilitating spontaneous breathing through the nasal route in normal humans sedated with midazolam.

Objectif : Déterminer l'effet de la mobilisation antérieure de la mandibule (MM) sur la respiration nasale spontanée chez des volontaires sains qui reçoivent du midazolam par voie intraveineuse.

Méthode : Chez quatre sujets qui présentaient une respiration nasale pendant la sédation avec du midazolam (dose intraveineuse : 0,09 $\pm 0,02 \mathrm{mg} \cdot \mathrm{kg}^{\prime}$, moyenne \pm écart type), nous avons mesuré la fréquence respiratoire $(F R)$, la vitesse maximale de l'écoulement d'air inspiratoire nasal $\left(\dot{\vee}_{\text {maxln }}\right)$, la vitesse maximale de l'écoulement d'air expiratoire nasal $\left(\dot{V}_{\text {maxEn }}\right)$, le coefficient de durée $(T i / T t o t)$ et la résistance nasale $(R n)$ avant et après la $M M$. On obtient la résistance nasale en divisant la pression différentielle masque nasal-oropharynx par la vitesse d'écoulement de l'air lors de l'inspiration nasale maximale.

Résultats: La FR, la $\dot{V}_{\max I n}$, et la $\dot{V}_{\max E n}$ ont augmenté après la $M M(P<0,00 \mathrm{l}$, respectivement). Par contre, Ti/Ttot a diminué après la MM $(P<0,001)$. En conséquence, la MM a réduit la $R n$ de 30,4 \pm 40,8 à 5,0 \pm 5,6 $\left(\mathrm{cmH}_{2} \mathrm{O} \cdot \mathrm{I}^{-1} \cdot \mathrm{s}^{-1}\right)$ (moyenne \pm écart type) $(P<0,00 \mathrm{I})$. Chez les quatre sujets, aucun écoulement de l'air par voie orale n'a été noté avant et après la $M M$.

Conclusion : La mobilisation antérieure de la mandibule réduit la résistance nasale et facilite ainsi la respiration nasale spontanée chez des humains normaux qui reçoivent du midazolam.

From the Departments of Anesthesiology, School of Medicine, Tokyo Medical and Dental University, ${ }^{*}$ Tokyo, Gifu University School of Medicine, $†$ Gifu and the Institute of Clinical Medicine, $\ddagger$ University of Tsukuba, Ibaraki, Japan.

Address correspondence to: Tsutomu Oshima MD, Department of Anesthesiology and Critical Care Medicine, Gifu University School of Medicine, 40 Tsukasamachi, Gifu-City, Gifu 500-8705, Japan. Phone: 81-58-267-2295; Fax: 81-58-267-2961;

E-mail: oshimat@cc.gifu-u.ac.jp

Accepted for publication November 28, 1999 
M IDAZOLAM increases upper airway resistance. ${ }^{1}$ We have recently shown that midazolam impairs nasal airway patency in normal humans. ${ }^{2}$ On the other hand, advancement of the mandible (ADM) increases the cross-sectional area of the velopharynx, that portion of the nasopharynx bounded ventrally by the soft palate, during wakefulness, ${ }^{3}$ during midazolam-induced sedation ${ }^{4}$ and during general anesthesia and total muscle paralysis. ${ }^{5}$ If velopharyngeal patency is improved by ADM, nasal breathing should be facilitated. However, there are no data regarding the effects of ADM on the mechanical parameters of spontaneous breathing through the nasal route in humans sedated with midazolam. We hypothesized that ADM would reduce nasal resistance, thereby facilitating spontaneous breathing through the nasal route during midazolam sedation. Our study was performed to test this hypothesis in normal human subjects.

\section{Methods}

The study protocol was approved by the Ethics Committee of Tokyo Medical and Dental University. Five male and three female volunteers, aged 25 to 33 yr, participated in the study. After obtaining written informed consent from each subject, we conducted a history and physical examination. Exclusion criteria were clinical evidence of nasal, pulmonary, cardiac, or CNS disease, obesity, use of centrally acting medications within the previous week, a history of smoking, sleep disordered breathing, recent upper respiratory infections, drug or alcohol abuse. Subjects refrained from oral intake for eight hours, and from alcohol and caffeine for $24 \mathrm{hr}$ before the study.

Each subject had one nasal passage anesthetized with $0.05 \mathrm{ml}$ lidocaine $8 \%$ spray. No nasal decongestants were used. Oropharyngeal pressure (Pop) was measured using a balloon catheter (Millar, od 1.7 $\mathrm{mm}$ ) introduced through the anesthetized nostril. The tip of the catheter was located just caudal to the inferior margin of the soft palate and rostral to the tongue in the oropharynx. The position was verified by direct visual examination of the posterior oropharynx. No migration of the oropharyngeal balloon catheter was observed during the study.

An airtight custom-made, partitioned face mask (Senkousha, Japan $)^{1,6,7}$ was fitted while the subject rested in the supine position. A hard rubber septum separated the mask into nasal and oral chambers, each with its own port (i.d. $9 \mathrm{~mm}$, length $40 \mathrm{~mm}$ ) and a side-arm outlet (i.d. $3.5 \mathrm{~mm}$ ). The rubber septum was positioned above the upper lip, so that the mouth and lips were kept slightly apart. Each mask chamber was tested separately for air leaks by pressurizing to 10 $\mathrm{cmH}_{2} \mathrm{O}$ and ensuring that the pressure was constant for $10 \mathrm{sec}$. The dead space for nasal and mouth chambers was 90 and $120 \mathrm{ml}$, respectively. Nasal airflow rate $(\dot{\mathrm{V}} \mathrm{n})$, nasal mask pressure $(\mathrm{Pnm})$, and Pop were monitored with a C-P pulmonary monitor (model CP 100, BICORE monitoring system, USA) connected to the breathing circuit. Oral airflow rate $(\mathrm{Vo})$ was measured using a Fleisch No. 2 pneumotachograph.

All measurements were continuously recorded on a paper chart recorder (model WS-800R, Nihon Kohden, Japan), and stored on a tape recorder (model XR-5000, TEAC, USA). To avoid any confounding information from upper airway anesthesia, we waited for $30 \mathrm{~min}$ after application of topical anesthesia before starting measurements.

\section{Experimental protocol}

After inserting a 22-Gauge catheter in the left cephalic vein, an intravenous infusion of acetated Ringer's solution was started at $100 \mathrm{ml} \cdot \mathrm{hr}^{-1}$. Oxygen saturation, ECG, and noninvasive blood pressure measurements were recorded.

After determining baseline respiratory measurements, we began midazolam administration: 0.01 $\mathrm{mg} \cdot \mathrm{kg}^{-1}$ by rapid (< $\left.1 \mathrm{sec}\right)$ intravenous injection, administered every minute, until subjects reached a consciousness level where they exhibited spontaneous eye closure and responded only to prodding or shaking. The midazolam dose required to attain this sedation ranged from 0.06 to $0.12 \mathrm{mg} \cdot \mathrm{kg}^{-1}$, with a mean of $0.09 \pm 0.02($ mean $\pm \mathrm{SD}) \mathrm{mg} \cdot \mathrm{kg}^{-1}$.

Four of the eight subjects exhibited snoring with oronasal airflow following midazolam administration. To simplify data analysis, they were excluded from further participation in the study. The other four subjects, consisting of three men and one woman, continued breathing through the nasal route during sedation with midazolam. In these four subjects, the mandible was manually displaced anteriorly by one of the authors. Maximal mandibular advancement was achieved with a two-handed technique.

Respiratory rate (RR), peak nasal inspiratory airflow rate $\left(\dot{\mathrm{V}}_{\text {nIpeak }}\right)$, peak nasal expiratory airflow rate $\left(\dot{\mathrm{V}}_{\text {nEpeak }}\right)$, the duty ratio (Ti/Ttot) and the nasal resistance ( $\mathrm{Rn})$ were obtained from 10 consecutive breaths before and after ADM. Ti/Ttot was calculated by dividing the duration of inspiration by total duration of each respiratory cycle. Rn was calculated by dividing the pressure difference between Pnm and Pop by airflow rate at $\dot{\mathrm{V}}_{\text {nIpeak }}$. To determine how these five respiratory parameters changed after ADM, two tailed paired t test was used. A significance level of 0.05 was used. 


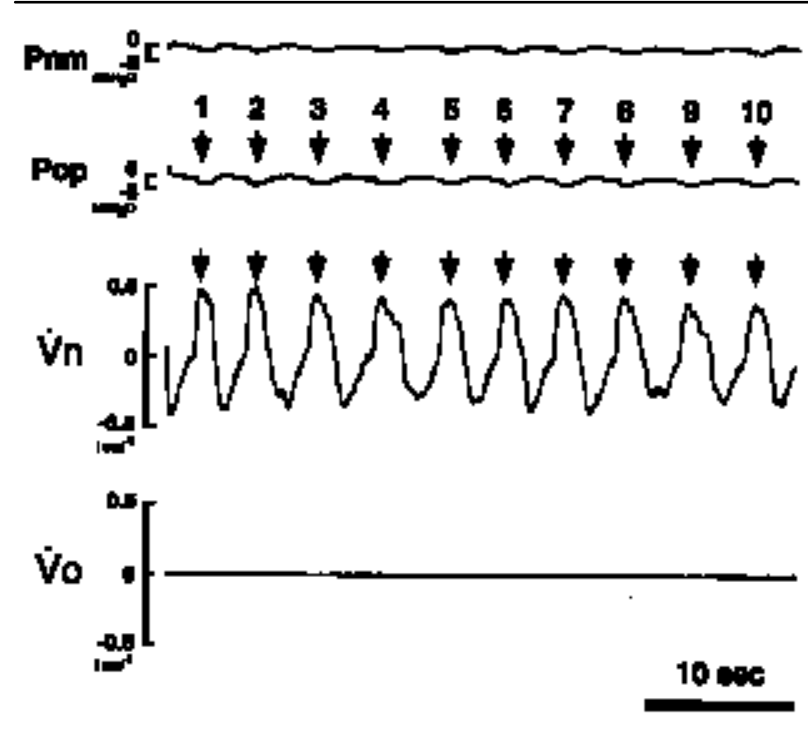

FIGURE I Representative record during wakefulness Vn increased proportionally to the negative pressure swing of Pop within each inspiration (breaths 1-10), exhibiting a round contour Each arrow indicates the time point when peak nasal airflow occurred within each inspiration. Pnm = nasal mask pressure $\left(\mathrm{cmH}_{2} \mathrm{O}\right)$, Pop $=$ oropharyngeal pressure $\left(\mathrm{cmH}_{2} \mathrm{O}\right)$; Nasal $(\mathrm{V} \mathrm{n})$ and oral airflow rate $(\mathrm{Vo})\left(1 \cdot \mathrm{sec}^{-1}\right)$ (See text for detail)

Results

Four subjects who underwent advancement of the mandible $(\mathrm{ADM})$ consisted of three men and one woman. Mean $( \pm \mathrm{SD})$ height and weight was $170.0 \pm$ $5.8 \mathrm{~cm}$ and $61.0 \pm 8.3 \mathrm{~kg}$, respectively. As demonstrated by low BMI $\left(20.9 \pm 2.0 \mathrm{~kg} \cdot \mathrm{m}^{-2}\right.$, mean $\left.\pm \mathrm{SD}\right)$, none were obese. Before midazolam administration, $\dot{\mathrm{V}} \mathrm{n}$ increased proportionally to the negative pressure swing of Pop within each inspiration (breaths 1-10), exhibiting a round contour (Figure 1). Figure 2 shows a representative change in spontaneous breathing pattern before and after ADM. During midazolam-induced sedation, spontaneous breathing was characterized by inspiratory airflow limitation accompanied by enhanced negative pressure swing of Pop (breaths 1-10). Immediately after ADM, the pressure swing of Pop decreased and both peak nasal inspiratory $\left(\dot{\mathrm{V}}_{\text {nIpeak }}\right)$ and expiratory airflow rate $\left(\dot{\mathrm{V}}_{\text {nEpeak }}\right)$ increased (breaths 11 21). Respiratory rate increased following ADM. There was no respiratory airflow through the oral route before and after ADM.

In the four subjects, respiratory rate $(\mathrm{RR}), \dot{\mathrm{V}}_{\text {nIpeak }}$ and $\dot{\mathrm{V}}_{\text {nEpeak }}$ increased $(P<0.001)$ (Table). Conversely, the duty ratio decreased following ADM $(P<0.001)$ (Table). Overall, the nasal resistance $(\mathrm{Rn})$ decreased

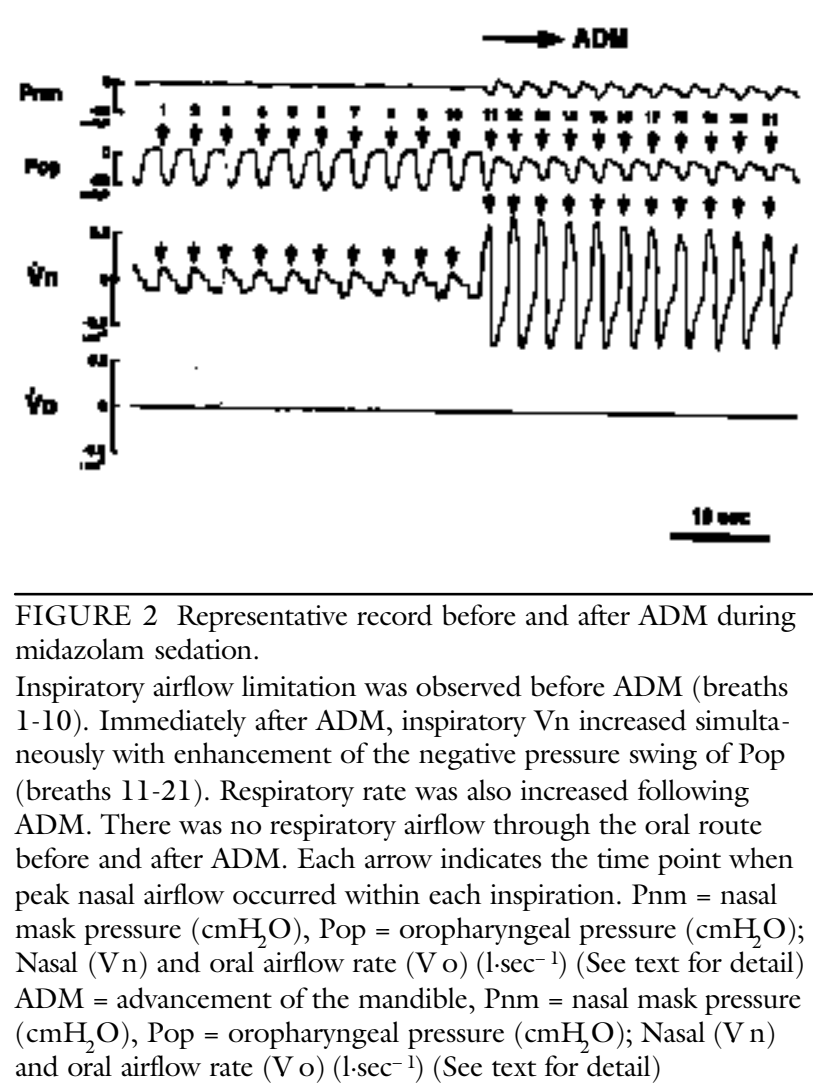

from $30.4 \pm 40.8$ to $5.0 \pm 5.6\left(\mathrm{cmH}_{2} \mathrm{O} \cdot \mathrm{l}^{-1} \cdot \mathrm{sec}^{-1}\right)$ $($ mean $\pm \mathrm{SD})(P<0.001)$ (Figure 3$)$. In these four subjects, no respiratory airflow was observed through the oral route before and after ADM.

\section{Discussion}

The present study showed that advancement of the mandible (ADM) rapidly decreases nasal resistance $(\mathrm{Rn})$, thereby facilitating spontaneous breathing through the nasal route in normal human subjects sedated with midazolam.

We have shown previously that midazolam impairs both nasal patency and the ability to change the breathing route in response to nasal airway obstruction. ${ }^{1}$ On the other hand, several investigators have previously demonstrated that ADM increases crosssectional area of the velopharynx in patients with OSA. ${ }^{2-4}$ Furthermore, anterior mandible protruding devices are known to be effective in treating OSA. ${ }^{8}$ However, there were no data concerning acute effects of ADM on spontaneous breathing pattern through the nasal route in normal human subjects sedated with midazolam. 
Since the tip of the oropharyngeal catheter was located just below the inferior margin of the soft palate, anatomical structures associated with $\mathrm{Rn}$ included the nasal passage from the inferior margin of the soft palate. Inspiratory airflow limitation, which sometimes occurred during midazolam-induced sedation (Figure 2), suggests partial collapse of the nasal airway segment related to $\mathrm{Rn}$. This partial collapse should allow airflow to increase with the negative pressure swing of Pop only up to a critical value for the transmural pressure (Pcrit). ${ }^{9}$ At more negative Pop, greater collapse occurs and there is no further increase in airflow despite greater inspiratory effort. This characteristic behaviour of the airway segment has been described as flow limitation. ${ }^{10}$

Following ADM, $\dot{\mathrm{V}}_{\text {nIpeak }}$ increased proportionally to the negative pressure swing of Pop, resulting in a round contour during each inspiration (Figure 2). This change in the contour of the inspiratory airflow tracing suggests a decrease in inspiratory UA resistance. ${ }^{11}$ In fact, a decrease in Rn occurred after ADM (Figure 3). These findings indicate that $\mathrm{ADM}$ improves nasal patency.

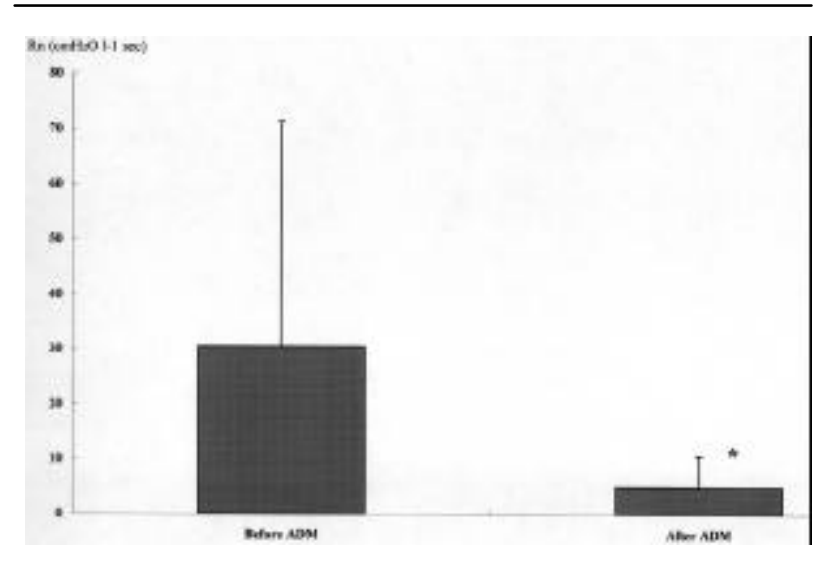

FIGURE 3 Nasal resistance $(\mathrm{Rn})\left(\mathrm{cmH}_{2} \mathrm{O} \cdot \mathrm{r}^{-1} \cdot \mathrm{sec}\right.$, mean $\left.\pm \mathrm{SD}\right)$ before and after ADM.

Rn significantly decreased from $30.4 \pm 40.8$ to $5.0 \pm 5.6$

$\left(\mathrm{cmH}_{2} \mathrm{O} \cdot 1^{-1} \cdot \mathrm{sec}\right)($ mean $\pm \mathrm{SD})(P<0.001)$.

${ }^{*} P<0.001$ compared with before ADM

$\mathrm{ADM}=$ advancement of the mandible (See text for detail)
Although the mechanism of action remains to be determined, two general modes of action are possible 1) ADM could increase the calibre of the passive velopharynx, and/or 2) ADM could activate upper airway dilator muscles. The first possibility has been verified in patients with OSA ${ }^{5}$ and in normal human subjects. ${ }^{12,13}$ Since $\mathrm{Rn}$ was used as indirect index of upper airway patency in the present study, the decrease in Rn may be consistent to this hypothesis.

With regard to the second possibility, we have recently demonstrated, using a remotely controlled mandibular positioner, that mandibular advancement decreases upper airway dilator muscle activity during sleep in patients with OSA. ${ }^{14}$ This device enables us to minimize the physical stimuli when the subject's mandible is protruded. ${ }^{4,14}$ However, the subject's mandible was manually advanced in the present study. The subject is more likely to be aroused by manual ADM, thereby activating upper airway dilator muscle. Further studies are needed to elucidate the role of upper airway dilator muscle during manual ADM.

The increase in RR, $\dot{\mathrm{V}}_{\text {nIpeak }}$, and $\dot{\mathrm{V}}_{\text {nEpeak }}$ observed in the present study may also be related to a brief arousal from sedation. On the other hand, a decrease in $\mathrm{Ti} / \mathrm{Ttot}$ may be in part caused by the decrease in Rn. As shown in Figure 2, ADM decreased the pressure swing of Pop and increased $\dot{V}_{\text {nEpeak }}$. These findings suggest that ADM may also decrease expiratory nasal resistance. To discuss this possibility, however, more information is necessary regarding the effects of midazolam on expiratory resistance along various segments of the total upper airway.

In the present study, no respiratory airflow was observed through the oral route before and after ADM. Sedation produced by thiopental ${ }^{7}$ and midazo$\mathrm{lam}^{1}$ suppresses the ability to change the breathing route. ADM is unlikely to facilitate oral breathing in normal human subjects sedated with midazolam.

Our partitioned face mask is the same as that used by Nishino and coworkers, which enables measurement of nasal and oral airflow separately. 1,6,7 Clearly, this mask produces a somewhat artificial situation with a considerable pressure exerted on the orofacial

TAB LE Changes in respiratory measurements before and after ADM Mean $\pm \mathrm{SD}$ for $\dot{\mathrm{V}}_{\text {nIpear }} \dot{\mathrm{V}}_{\text {nEpear }} \mathrm{Ti} /$ Ttot; Median for $\mathrm{RR}$ ${ }^{*} P<0.001$ compared with before ADM

$\mathrm{ADM}=$ advancement of the mandible, $\dot{\mathrm{V}}_{\mathrm{nIpeak}}=$ peak nasal inspiratory airflow rate $\left(1 \cdot \mathrm{sec}^{-1}\right), \dot{\mathrm{V}}_{\mathrm{nEpeak}}=$ peak nasal expiratory airflow rate $\left(1 \cdot \sec ^{-1}\right), \mathrm{RR}=$ respiratory rate $\left(\mathrm{min}^{-1}\right), \mathrm{Ti} / \mathrm{Tt}$ tot $=$ duty ratio

\begin{tabular}{lllll}
\hline & $\dot{\mathrm{V}}_{\text {nIpeak }}\left(1 \cdot \mathrm{sec}^{-1}\right)$ & $\dot{\mathrm{V}}_{\text {nEpeak }}\left(1 \cdot \mathrm{sec}^{-1}\right)$ & $\mathrm{RR}\left(\mathrm{min}^{-1}\right)$ & $\mathrm{Ti} / \mathrm{Ttot}$ \\
Before ADM & $0.21 \pm 0.06$ & $0.19 \pm 0.05$ & 15 & $0.52 \pm 0.12$ \\
After ADM & $0.52 \pm 0.12^{*}$ & $0.45 \pm 0.10^{*}$ & $19^{*}$ & $0.40 \pm 0.08^{*}$ \\
\hline
\end{tabular}


region. This artificial environment may affect the results of our study. Furthermore, a final limitation of the present study is the small number of subjects showing effects of ADM on spontaneous breathing through the nasal route. To simplify data analysis, four subjects who exhibited snoring and/or oronasal breathing during midazolam-induced sedation were excluded from further participation in the study. Confirmation of these observations in a large number of subjects is necessary.

We conclude that ADM decreases nasal resistance, thereby facilitating spontaneous breathing through the nasal route in human subjects sedated with midazolam.

Acknowledgment

The authors are grateful to Dr. T. Sawa for technical support.

\section{References}

1 Montravers P, Durenil B, Demonts JM. Effects of i.v. midazolam on upper airway resistance. $\mathrm{Br} \mathrm{J}$ Anaesth 1992; 68: 27-31.

2 Oshima T, Masaki $\Upsilon$, Toyooka H. Flumazenil antagonizes midazolam-induced airway narrowing during nasal breathing in humans. Br J Anaesth 1999; 82: 698-702.

3 Ferguson KA, Love LL, Ryan CF. Effects of mandibular and tongue protrusion on upper airway size during wakefulness. Am J Respir Crit Care Med 1997; 155: 1748-54.

4 Oshima T, Vazquez JC, Masuda A, Tsai WH, Hajduk E, Remmers JE. Effects of incremental mandibular protrusion on cross-sectional area of the passive pharynx during medicated sleep in patients with patients with obstructive sleep apnea (OSA). Am J Respir Crit Care Med 1997; 155: A676.

5 Isono S, Tanaka A, Sho $\Upsilon$, Konno A, Nishino T. Advancement of the mandible improves velopharyngeal airway patency. J Appl Physiol 1995; 79: 2132-8.

6 Nishino T, Sugiyama A, Tanaka A, Ishikawa T. Effects of nasal anaesthesia on shift of breathing route in adults. Lancet 1992; 339: 1497-500.

7 Nishino T, Kochi T. Effects of sedation produced by thiopentone on responses to nasal occlusion in female adults. Br J Anaesth 1993; 71: 388-92.

8 O'Sullivan RA, Hillman DR, Mateljan R, Pantin C, Finucane KE. Mandibular advancement splint: an appliance to treat snoring and obstructive sleep apnea. Am J Respir Crit Care Med 1995; 151: 194-8.

9 Smith PL, Wise RA, Gold AR, Schwartz AR, Permutt S. Upper airway pressure-flow relationships in obstructive sleep apnea. J Appl Physiol 1988; 64: 789-95.
10 Dawson SV, Elliott EA. Wave-speed limitation and expiratory flow - a unifying concept. J Appl Physiol: Respir Environ Exer Physiol 1977; 43: 498-515.

11 Condos R, Norman RG, Krishnasamy I, Peduzzi N, Goldring RM, Rapoport DM. Flow limitation as a noninvasive assessment of residual upper-airway resistance during continuous positive airway pressure therapy of obstructive sleep apnea. Am J Respir Crit Care Med 1994; 150: 475-80.

12 Isono $S$, Tanaka A, Tagaito $\Upsilon$, Sho $\Upsilon$, Nishino $T$. Pharyngeal patency in response to advancement of the mandible in obese anesthetized persons. Anesthesiology 1997; 87: 1055-62.

13 Reber A, Wetzel SG, Schnabel K, Bongartz G, Frei FJ. Effect of combined mouth closure and chin lift on upper airway dimensions during routine magnetic response imaging in pediatric patients sedated with propofol. Anesthesiology 1999; 90: 1617-23.

14 Oshima T, Tsai WH, Hajduk E, Remmers JE. Mandibular protrusion decreases genioglossal EMG during sleep in patients with obstructive sleep apnea. Am J Respir Crit Care Med 1998; 157: A655. 